\title{
Respiratory Motoneuronal Activity Is Altered by Injections of Picomoles of Glutamate into Cat Brain Stem
}

\author{
Donald R. McCrimmon, Jack L. Feldman, and Dexter F. Speck' \\ Departments of Physiology and Anesthesia, Northwestern University, Chicago, Illinois 60611
}

The local neural circuitry underlying the control of breathing was studied by injecting nanoliter volumes of excitatory amino acids into discrete regions of cat brain stem. Experiments were performed on chloralose-urethane anesthetized, vagotomized, paralyzed, and artificially ventilated cats. Phrenic, intercostal, and recurrent laryngeal nerve discharges were recorded. Multibarrel pipettes were used for recording and pressure ejection of drugs or a dye for marking recording and ejection sites. Ejected volumes were directly monitored for every injection. Injections, proximal to neurons discharging with a respiratory periodicity, of as little of $200 \mathrm{fmol}$ of $\mathrm{L}$-glutamate in $200 \mathrm{pl}$ of saline elicited marked, site-specific increases or decreases in respiratory motoneuronal discharge. $N$-Methyl-D-aspartic acid and homocysteic acid elicited similar site-specific alterations in respiratory motor output, although some details of the response could differ qualitatively. Responses to all the excitatory agents used were attenuated by concurrent injection of kynurenic acid, DL-2-amino-4-phosphonobutyric acid, or glutamic acid diethyl ester. There was no change in spontaneous phrenic nerve discharge in response to injections of equivalent or larger volumes of saline or lidocaine. These results indicate a heterogeneity in the spatial organization of the brain-stem neural circuitry underlying respiratory control, which has not been described previously. This injection technique may provide a mechanism for probing the neural circuitry underlying other behaviors.

Brain function underlying behavior in mammals involves information transfer between neurons in complex networks. The nuclear and layered structure of the mammalian brain suggests that small aggregates of neurons act as functional units. It is of fundamental interest to determine the nature of this local information transfer and its consequences in terms of behavior. In this paper, we present an approach to study this problem using site-specific delivery of (sub)picomolar amounts of excitatory amino acids in nanoliter volumes. Using this approach, we present evidence of a neuronal mosaic underlying the control of breathing.

Neurons that discharge with a respiratory periodicity are concentrated in the nucleus ambiguus-retroambigualis (ventral respiratory group, VRG) and the nucleus tractus solitarius of the medulla (Cohen, 1979; Feldman, in press; von Euler, 1983).

Received Oct. 24, 1985; revised Jan. 21, 1986; accepted Jan. 22, 1986.

The technical assistnce of Paul G. Burns and the helpful comments of Professor Oscar Hechter are gratefully acknowledged. We would like to thank Drs. Howard H. Ellenberger, Debra E. Weese-Mayer, and Jeffery C. Smith for their help in experiments at the latter stages of this study. This work was supported by NIH Grant NS-21036.

Correspondence should be addressed to Jack L. Feldman, Department of Kinesiology, University of California, Los Angeles, 405 Hilgard Avenue, Los Angeles, CA 90024 .

Present address: Department of Physiology and Biophysics, A. B. Chandler Medical School, University of Kentucky, Lexington, KY 40504.

Copyright (c) 1986 Society for Neuroscience $0270-6474 / 86 / 082384-09 \$ 02.00 / 0$
The VRG extends rostrally from the spinomedullary junction to the level of the retrofacial nucleus. Neurons within the VRG can be divided into 2 populations based on their impulse discharge pattern. Neurons that produce bursts of action potentials synchronous with the phrenic nerve discharge (inspiratory neurons) are concentrated between the obex and most rostral portion of the VRG - the Bötzinger complex (Kalia et al., 1979; Lipski and Merrill, 1980; Merrill and Fedorko, 1984). Neurons that produce bursts of impulse activity during the periods of phrenic nerve silence (expiratory neurons) are located between the obex and the upper cervical spinal cord and in the Bötzinger complex. (Vagal motoneurons are concentrated in the nucleus ambiguus.) Both the inspiratory and expiratory neuronal populations contain bulbospinal neurons and intrinsic brain-stem interneurons. Although the gross spatial organization of the VRG is well established, the fine structure of the underlying network has not been elucidated. To study the local organization and pharmacology of the VRG, we developed a technique for making extremely small $(<0.2-10 \mathrm{nl})$ injections of dilute solutions (typically 1-10 $\mathrm{mM}$ ) of excitatory amino acids into electrophysiologically and histologically confirmed regions of the VRG.

\section{Materials and Methods}

Experiments were performed on 20 adult cats anesthetized with chloralose-urethane $(40$ and $250 \mathrm{mg} / \mathrm{kg}$, i.v., respectively, and supplemented as required-see Feldman et al., 1984). Atropine methyl nitrate $(0.2$ $\mathrm{mg}$, i.m.) was given prior to beginning surgery to prevent secretions. Cannulas were inserted into a femoral artery and vein and into the trachea. Cats were placed in a rigid head holder, paralyzed with gallamine triethiodide, thoracotomized bilaterally, and mechanically ventilated with $100 \%$ oxygen. Rectal temperature was maintained at about $38^{\circ} \mathrm{C}$ using a servo-controlled heating pad and heat lamp. The vagus and fifth cervical branches of the phrenic nerves were isolated bilaterally in the neck via a dorsal approach and sectioned distally. The phrenic nerves were mounted on bipolar silver wire electrodes for recording. In some cats the right recurrent laryngeal, right hypoglossal, or left seventh $\left(T_{7}\right)$ and tenth $\left(T_{10}\right)$ thoracic intercostal nerves (external and internal branches) were isolated, cut distally, and their central ends placed on bipolar silver hook recording electrodes. An occipital craniotomy was performed, the dura removed, and the caudal cerebellum reflected or aspirated to expose the brain stem.

End-tidal carbon dioxide was continuously monitored with a rapidly responding infrared analyzer and maintained at about $5 \%$ by adjusting the ventilator or by the addition of carbon dioxide into the inspired oxygen. Systemic arterial pressure was monitored from the femoral arterial catheter. Femoral arterial blood was sampled periodically and analyzed for partial pressures of carbon dioxide and oxygen and for acid-base status. The occurrence of a metabolic acidosis was corrected by infusing a $5 \%$ solution of sodium bicarbonate as required. Nerve electrodes were connected to high-impedance differential instrumentation amplifiers and the output fed in parallel to an oscilloscope, tape recorder, and phrenic nerve signal processor. The phrenic processor derived a gate marking the period of phrenic nerve discharge (neural inspiration), which was used to control the timing of drug injections (see below). At the end of each expcriment, the animal was deeply 


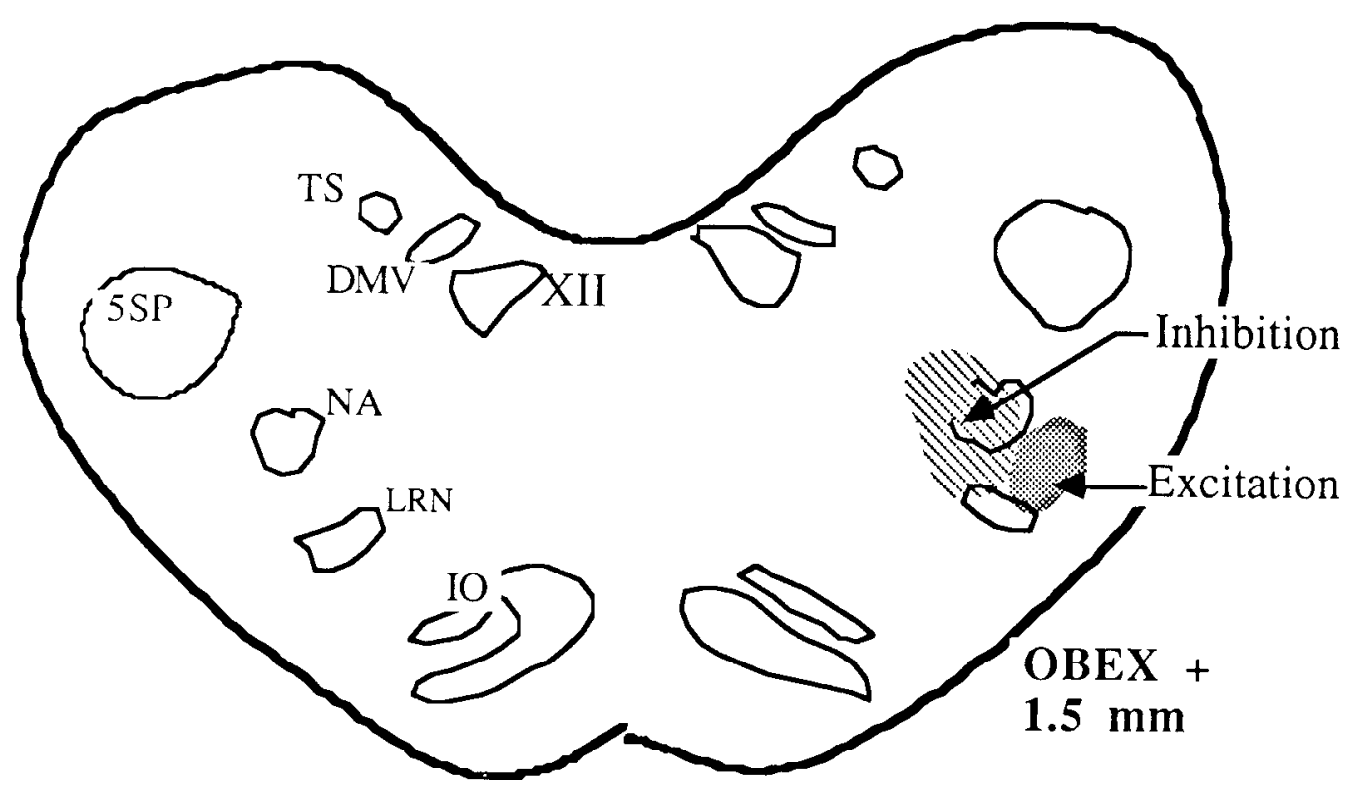

Figure 1. Reconstruction of medulla $1.5 \mathrm{~mm}$ rostral to obex showing regions from which injections of excitatory amino acids elicited increases (excitation; Figs. 2-4, 7) or decreases (inhibition; Figs. 5, 6) in phrenic motoneuronal discharge; selected injection sites were marked by the injection of pontamine sky blue. Structures are named according to Berman's atlas (1968). 5SP spinal trigeminal nucleus; $X I I$, hypoglossal nucleus; $D M V$, dorsal motor nucleus of the vagus; $L R N$. internal division of the lateral reticular nucleus; $N A$, nucleus ambiguus; $T S$, solitary tract.

anesthetized and perfused transcardially with $10 \%$ formalin. The brain stem was removed for subsequent histological verification of recordinginjection sites (LaBossiere, 1976).

One to 7 barrel micropipettes suitable for recording extracellular impulse activity and drug ejection were constructed with total external tip diameters of 3-12 $\mu \mathrm{m}$. Barrels contained 1, 10, or $100 \mathrm{~mm}$ Glu, $10 \mathrm{~mm}$ DL-homocysteic acid (DLH), $10 \mathrm{~mm} N$-methyl-D-aspartic acid (NMDA), $4 \%$ lidocaine hydrochloride, $1 \mathrm{~mm}$ kynurenic acid (Kyn), $100 \mathrm{~mm}$ glutamic acid diethyl ester (GDEE), $10 \mathrm{mM}$ DL-2-amino-4-phosphonobutyrate (APB), $100 \mathrm{~mm}$ GABA, $10 \mathrm{~mm}$ thyrotropin-releasing hormone (TRH), $10 \mathrm{~mm}$ carbamylcholine chloride (carbachol), $10 \mathrm{~mm}$ acetylcholine chloride $(\mathrm{ACh}), 2 \%$ pontamine sky blue, or $165 \mathrm{~mm} \mathrm{NaCl}$. Drugs of $100 \mathrm{~mm}$ concentration were prepared in $100 \mathrm{~mm} \mathrm{NaCl}$, pontamine was prepared in $0.5 \mathrm{M}$ sodium acetate, and all others were prepared in $165 \mathrm{mM} \mathrm{NaCl}$ and the $\mathrm{pH}$ adjusted to $\approx 7.4$ with $1 \mathrm{~N} \mathrm{NaOH}$. At selected target sites, measured volumes of drugs were injected with short-duration (10-1000 $\mathrm{msec}$ ) pressure (0.1-7 atmosphere) pulses. Volumes of drug ejected were directly measured by monitoring the movement of the meniscus in a pipette barrel with the aid of a $100 \times$ microscope equipped with a fine reticule. It has been suggested that a pulse of a given pressure and duration delivered to a pipette barrel will reliably and reproducibly eject a given drug volume in vivo (Dray et al., 1983; Palmer et al., 1980; Welzl et al., 1985). We found that the volume of ejectate could vary considerably as the pipette was moved through live brain tissue, presumably due to changes in flow resistance as the microcnvironment at the clcctrode tip varied. Thus, at any given site there was a monotonic relationship between the pressure-pulse time product and the volume ejected, but this relationship could vary considerably between sites as little as $100 \mu \mathrm{m}$ apart. It was therefore necessary to measure the volume of each injection directly.

\section{Experimental paradigm}

Preliminary mapping studies were carried out using 1 to 3 barrel micropipettes. One barrel always contained either $10 \mathrm{~mm}$ DLH or Glu. The second and third barrels contained pontamine sky blue and either saline or an antagonist. The micropipette was inserted into the medulla dorsal to the VRG and advanced until respiratory modulated impulse activity was recorded. When respiratory neurons were located, the electrode was moved to a position $200-400 \mu \mathrm{m}$ above this activity. At this site an injection of $0.2-10 \mathrm{nl}$ of Glu or DLH was made and the motoneuronal response recorded on analog tape. The electrode was advanced in 50-100 $\mu \mathrm{m}$ steps while recording neuronal impulse activity. The injection protocol was repeated every $200-300 \mu \mathrm{m}$ until a motoneuronal response was no longer detected and no respiratory activity had been recorded for at least $300 \mu \mathrm{m}$. The electrode was then removed from the brain stem and displaced $500 \mu \mathrm{m}$ in the horizontal plane (mediolaterally or rostrocaudally). This procedure was iterated until inspiratory neurons (in the VRG, from the obex to the Bötzinger complex) were no longer detected with further electrode movements. After several experiments, a preliminary map of the site-specific responses in the VRG was completed. In subsequent experiments, electrodes consisting of 4-7 barrels were used to characterize more fully the regions that gave significant responses. The aims of these studies were threefold. First, we wished to determine the glutamate-receptor-mediated component of the response and to determine the dose (volume and concentration) dependence of the responses. The response to injections at some sites had a rapid onset and offset with limited time course $(<1 \mathrm{sec})$, which permitted the delivery of brief injections timed to a particular part of the respiratory phase. Second, we wanted to compare the responses elicited by the excitatory amino acids with those elicited by TRH, ACh, or carbachol and to determine the susceptibility of the response to antagonism by known Glu receptor blocking agents. And third, we wanted to determine, by dye injection and subsequent histological examination, the region from which particular responses were elicited.

\section{Data analysis}

The effects of drug injection on the pattern of respiratory motoneuronal discharge were determined with the aid of a laboratory computer. Effects on the overall pattern of phrenic nerve discharge were evaluated by sampling the integrated phrenic nerve discharge at $300-500 \mathrm{~Hz}$ and converting it to 12-bit digital records (Speck and Feldman, 1982). The duration of (neural) inspiration and expiration and peak amplitude of integrated phrenic nerve activity were determined for respiratory cycles before, during, and after injections.

The injection of excitatory amino acids into discrete sites within the VRG elicited sterotypic increases or decreases in phrenic motoneuronal discharge. For convenience, the increases will be referred to as excitation and the decreases as inhibition, although it is recognized that these could represent disinhibition or disfacilitation.

\section{Results}

Injections of small volumes $(0.2-10 \mathrm{nl})$ of excitatory amino acids into discrete regions of the VRG elicited site-specific, highly reproducible changes in respiratory motor outflow (Figs. 1-7). Impulse activity of neurons in the vicinity of the electrode tip was always increased by the administration of $2 \times 10^{-13}$ to $10^{-7}$ 

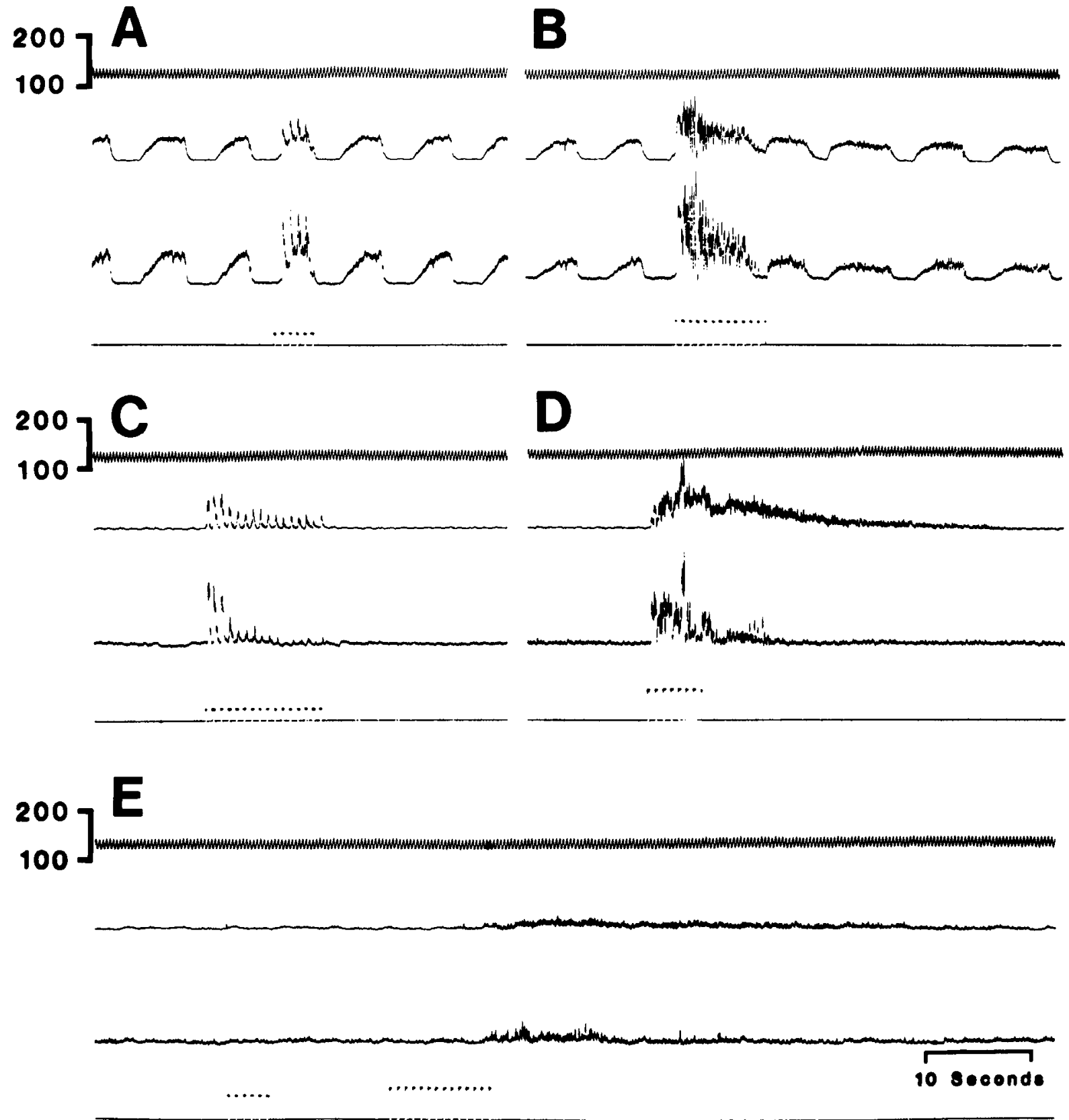

Figure 2. Excitation of phrenic motoneuronal activity produced by injections of Glu at a single site in the region of the ventral respiratory group $1 \mathrm{~mm}$ rostral to the obex in cat brain stem (excitatory region, Fig. 1). Recording-ejecting electrode was located in a region that contained only inspiratory-modulated neuronal activity. In each panel: top trace, blood pressure (mm Hg); second and third traces, ipsilateral (right) and contralateral (left) phrenic nerve activity integrated with a Paynter filter ( $80 \mathrm{msec}$ time constant); fourth trace, marker indicating the duration of a pressure pulse causing ejection of the Glu solution from the electrode. $A$, Glu, $1 \mathrm{mM}, 4 \mathrm{nl}$ total $(0.7 \mathrm{nl} / \mathrm{pulse}, 700 \mathrm{fmol} \mathrm{Glu} / \mathrm{pulse})$. Note that the first and last pulses, delivered during expiration, are relatively ineffective in eliciting a response. $B$, Glu, $10 \mathrm{~mm}, 4 \mathrm{nl}$ total $(0.5 \mathrm{nl} / \mathrm{pulse}, 5 \mathrm{pmol} / \mathrm{pulse}) . C-E$, Cat was hyperventilated to abolish rhythmic phrenic nerve activity. $C$, Glu, $1 \mathrm{~mm}, 4 \mathrm{nl}$ total $(0.2 \mathrm{nl} / \mathrm{pulse}, 200 \mathrm{fmol} / \mathrm{pulse})$. Attenuation of response may be due to periodic alteration in sensitivity due to underlying respiratory rhythm. Depolarization block is unlikely since higher concentrations of Glu elicited significantly greater responses (see $D)$. $D$, Glu, $100 \mathrm{~mm}, 2 \mathrm{nl}$ total $(0.25 \mathrm{nl} / \mathrm{pulse}, 25 \mathrm{pmol} / \mathrm{pulse}$ ). $E$, For $10 \mathrm{~min}$ prior to trace, 10 mM APB was injected at $20 \mathrm{nl} /$ minute. First set of pulses: $10 \mathrm{~mm}$ Glu, $4 \mathrm{nl}$ total; second set of pulses: $100 \mathrm{~mm}$ Glu, $4 \mathrm{nl}$ total.

mol Glu (e.g., Fig. 4), yet respiratory motor outflow could be augmented, depressed, or unaffected depending on the precise positioning of the electrode tip (Fig. 1). In the VRG, caudal to or more than $2.5 \mathrm{~mm}$ rostral to the obex, there was no discernible phrenic motoneuronal response to excitatory amino acid injection. Between the obex and $2.5 \mathrm{~mm}$ rostral to the obex in the VRG, we found 2 regions (verified histologically) from which phrenic motoneuronal inhibition or excitation could be elicited.
These regions were highly circumscribed, and the responses to excitatory amino acid injection were reproducible both within and between cats (Fig. 1). When ejections were made close to the boundary between the regions producing excitation or inhibition, a $200-300 \mu \mathrm{m}$ movement of the pipette could result in a qualitative change in the response. As the pipette was advanced out of the region from which respiratory neuronal impulse discharge could be recorded, the response to Glu ejection 

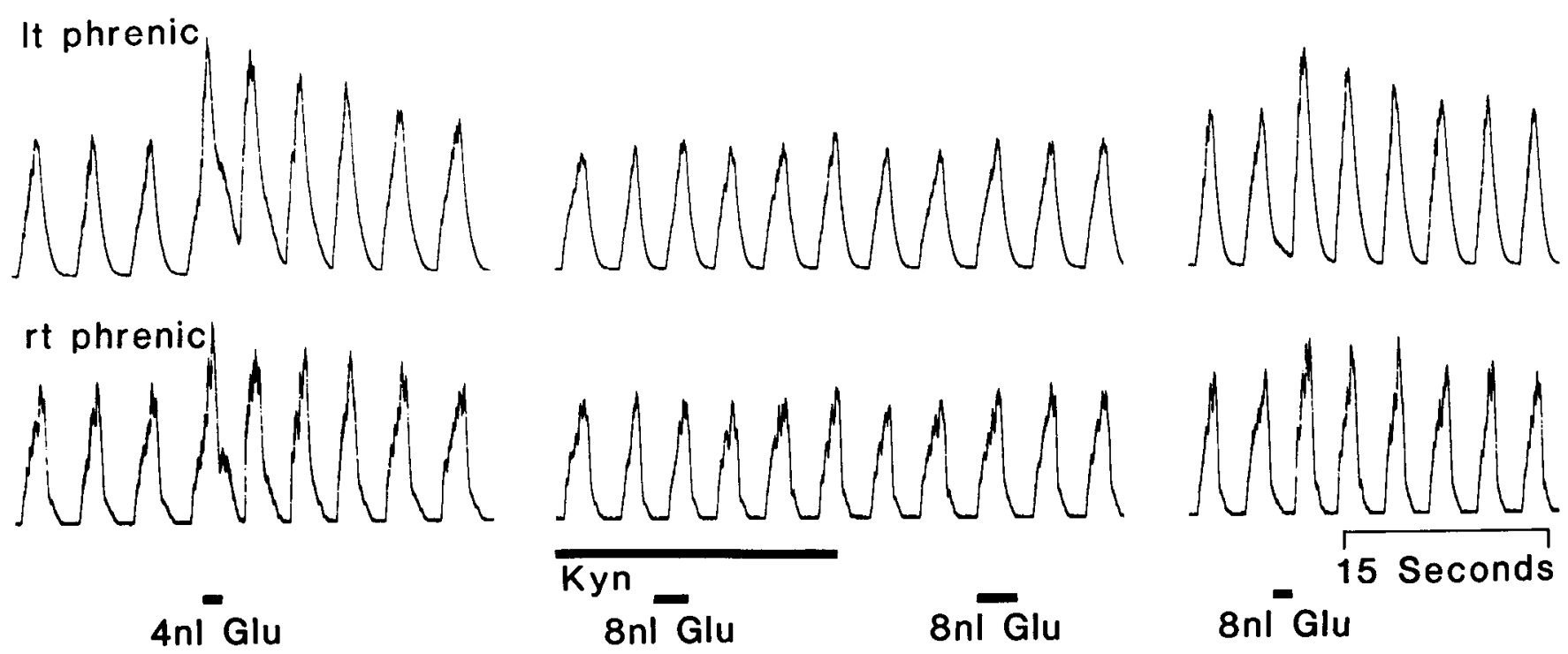

Figure 3. Kyn antagonism of Glu excitation of phrenic nerve discharge. Glu (10 mm) injected in the volumes indicated. Left, Glu, $4 \mathrm{nl}$, elicits bilateral increase in phrenic nerve discharge when injected $1.5 \mathrm{~mm}$ rostral and $4.0 \mathrm{~mm}$ lateral to the obex. Middle, Kyn, $1 \mathrm{~mm}(30 \mathrm{nl})$ injection (indicated by bar, injection was begun earlier than shown) abolished the responsc to $8 \mathrm{nl}$ Glu, an effect that outlasted the duration of the Kyn ejection. Right, Partial recovery of the excitatory response to Glu 40 min later. Note the slight reduction in the amplitude of phrenic nerve discharge during Kyn administration with subsequent recovery shown in the right panel.

rapidly attenuated and disappeared within $200-400 \mu \mathrm{m}$ of leaving the region. We did not explore regions beyond this immediate surround.

Potent, "concentration dependent" excitatory responses were obtained from a small region centered in the lateral VRG 1.5$2.0 \mathrm{~mm}$ rostral to the obex (Figs. 1-4). The typical concentration-dependent effects of Glu on respiratory motor outflow are shown in Figure 2. In response to injections of $<1 \mathrm{nl}$ of $1 \mathrm{~mm}$ Glu, there were marked transient increases in the amplitude of integrated phrenic motoneuronal discharge bilaterally (Fig. $2 \mathrm{~A}$, $C)$. Injections of higher concentrations of Glu altered the timing of phrenic motoneuronal bursting (Fig. $2 B$ ). These effects were blocked by concurrent injections of a Glu antagonist such as APB (Fig. $2 E$ ) or Kyn (Fig. 3). Movement of the electrode 200 $\mu \mathrm{m}$ or more in any direction (dorsoventral, rostrocaudal, mediolateral) from the location associated with the most potent response led to marked attentuation or loss of the excitatory response (Fig. 7).

NMDA produced a qualitatively different pattern of respiratory motor output excitation compared to Glu. As shown in Figure 4, 0.5-4 nl of $10 \mathrm{~mm}$ NMDA $\left(0.5\right.$ to $\left.4 \times 10^{-11} \mathrm{~mol}\right)$ produced a tonic discharge of phrenic motoneurons bilaterally. Superimposed on the tonic discharge were brief bursts of augmented discharge that occurred at the rate of 2-3 bursts/sec, a much greater frequency than the preinjection inspiratory burst frequency (Fig. 4). The duration of this NMDA response was considerably longer than the Glu response (compare Figs. 2 and 3 with Fig. 4). TRH, ACh, or the cholinergic agonist carbachol injected from separate barrels at the same site had no observable effect on phrenic nerve discharge. The injection of lidocaine into this region reduced the discharge of neurons in the vicinity of the electrode tip and prevented the neuronal and the motor nerve responses to either Glu or NMDA in a volume- (dose-) dependent manner but had no effect on spontaneous phrenic nerve discharge.

Depression of phrenic nerve discharge was elicited from a comparatively large portion of the VRG. Injections of $0.2-4.0$ $\mathrm{nl}$ of 1-10 mM Glu, DLH, or NMDA in the medial VRG between the obex and approximately $2.5 \mathrm{~mm}$ rostral produced a transient decrease in phrenic motoneuronal discharge (Fig. 5), as well as in expiratory motoneuronal activity recorded from both the contralateral $T_{7}$ or $T_{10}$ intercostal and the ipsilateral recurrent laryngeal motoneurons (Fig. 6). Injections timed to occur about $100 \mathrm{msec}$ prior to the expected onset of an inspiratory burst on the phrenic nerve could delay the onset of that burst (Fig. 5). The magnitude of these effects increased with increases in either concentration (range, 1-10 mM) or ejected volume (range, $0.2-4.0 \mathrm{nl}$ ). Qualitatively similar effects were obtained with Glu and DLH, although the time course of the response to DLH was slower in onset and longer lasting; a slower time course for DLH activation of single neurons is known to occur (Engberg et al., 1979a, b). NMDA produced a much more potent and longer-lasting depression of phrenic motoneuronal discharge than either Glu or DLH. Glu or DLH rarely abolished all rhythmic respiratory discharge on the phrenic nerve, whereas NMDA abolished respiratory related discharges for periods of up to 1 min when administered into this portion of the VRG. Kyn was particularly effective at blocking the responses to the excitatory amino acids (Fig. 3), while APB required relatively large volumes $(20-50 \mathrm{nl})$ to be effective (Fig. 2), possibly due to the partial agonist actions of L-APB at somc Glu receptors (Crunelli et al., 1985).

None of the above responses (or those described in the next paragraph) to excitatory amino acid injection was observed following ejection of equivalent volumes of saline, lidocaine, GABA, or any of the Glu antagonists. All responses to the excitatory amino acids were blocked by concurrent ejections of Kyn, GDEE, or APB (Figs. 2, 3).

\section{Blood pressure effects}

Within a region of the ventrolateral medulla from which exclusively respiratory neuronal impulse activity was recorded, ejection of Glu, DLH, or NMDA elicited rapid-onset decreases in arterial pressure with little or no decrease in heart rate (e.g., from $165 / 135$ to $75 / 45 \mathrm{~mm} \mathrm{Hg}$ and from 212 to 206 beats $/ \mathrm{min}$; Fig. 7). Since animals were vagotomized, activation of vagal preganglionic motoneurons could not elicit the decrease in heart rate. The region from which depressor responses were elicited was highly localized but overlapped both the region producing a decrease, as well as the region eliciting an increase, in phrenic 

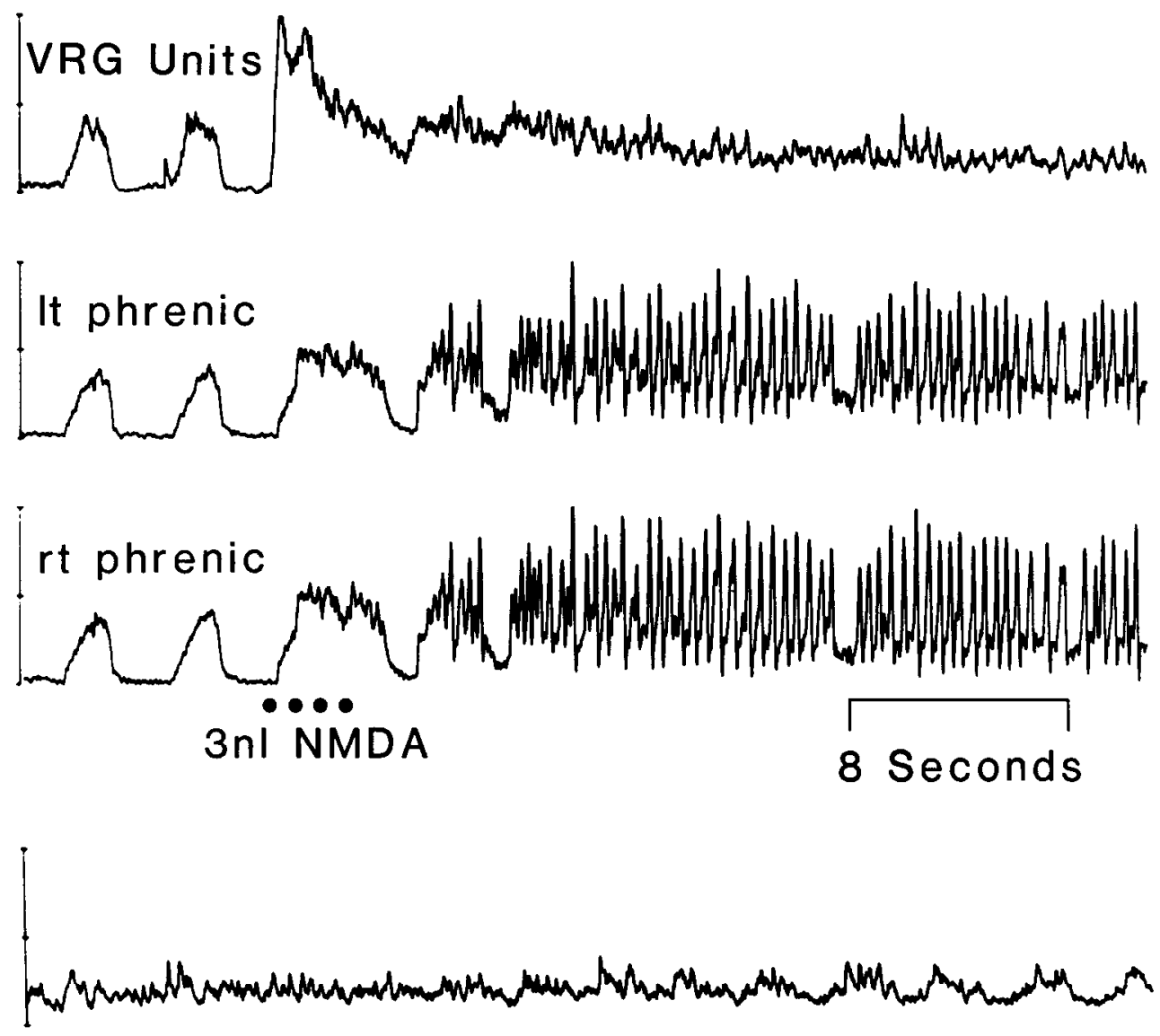

Figure 4. Response of phrenic nerve discharge to $10 \mathrm{~mm}$ NMDA injected into the excitatory region of the VRG (Fig. 1). Top trace, Integrated unit discharge recorded from the electrode used to eject the NMDA; next two traces, integrated left and right phrenic nerve discharge; bottom triplet of traces, continuation of top triplet. A total of $3 \mathrm{nl}$ NMDA ( $10 \mathrm{~mm}$ ) was injected in 4 pulses (700 pl/pulse) indicated by the dots. Note the "bursting" pattern of discharge elicited by NMDA and the long duration of the response. Scale bars are in arbitrary units.

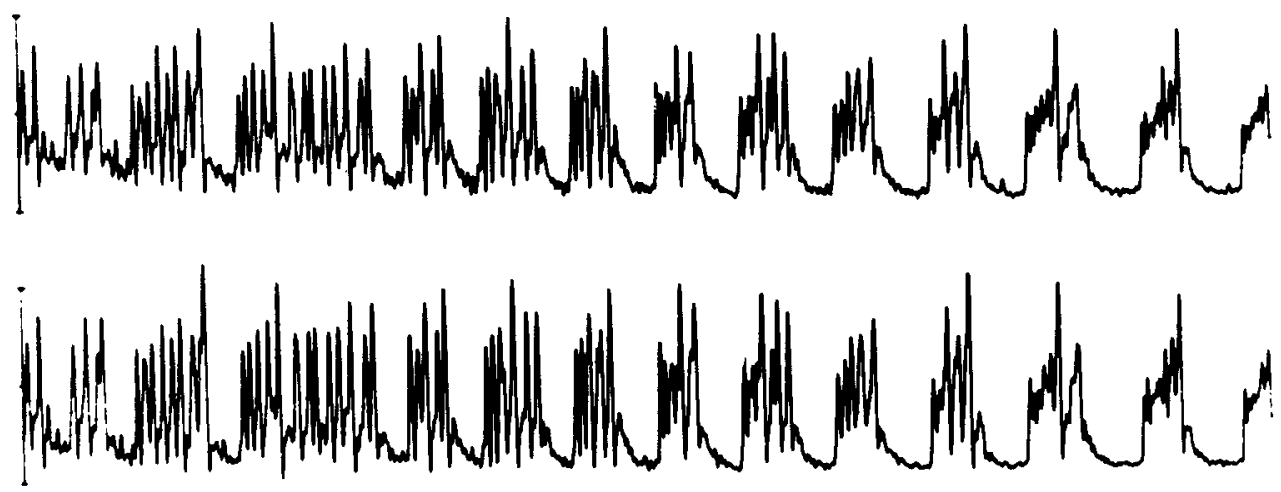

motoneuronal discharge. Nonetheless, areas from which cardiovascular or respiratory responses could be elicited were not identical. There were regions from which excitatory amino acids elicited marked increases or decreases in phrenic nerve discharge without any observable change in arterial pressure; the region eliciting depressor responses was not fully mapped but extended ventrolateral to those regions from which changes in respiratory motoneuronal discharge could be elicited.

\section{Discussion}

We have shown that injection of as little as $200 \mathrm{fmol}$ of an excitatory amino acid into a discrete region of the brain stem, the VRG, can elicit marked changes in a fundamental motor synergy, respiratory motor outflow. In addition, these localized injections demonstrate a heterogeneous spatial micro-organization of the VRG that may be an important factor underlying the neural control of respiration.

The action of the excitatory amino acids is likely to be due to increased discharge of neurons with Glu receptors on somatodendritic membranes, but not axons of passage, in the immediate vicinity of the electrode tip (Curtis and Ryall, 1966; Goodchild et al., 1982; Zieglgansberger and Puil, 1973). A mechanism of action via the activation of Glu receptors is supported by the ability of relatively selective Glu receptor blocking agents to antagonize the responses. Other nonspecific mechanisms of action are unlikely, since $\mathrm{ACh}$, carbachol, and TRHagents known to have stimulatory effects on breathing (Dev and Loeschcke, 1979; Hedner et al., 1983) and single respiratory neurons (Bradley and Lucy, 1983; Dekin et al., 1985; MorinSurun et al., 1984) - had no effect on respiratory motoneuronal discharge when administered at the sites from which Glu elicited peak increases or decreases in phrenic nerve discharge. Nevertheless, 2 possible sources of artifact must be considered: (1) pressure artifacts, which are ruled out by the absence of an effect of ejections of equivalent or larger volumes of saline or an antagonist, and (2) depolarization block (Crawford and Curtis, 


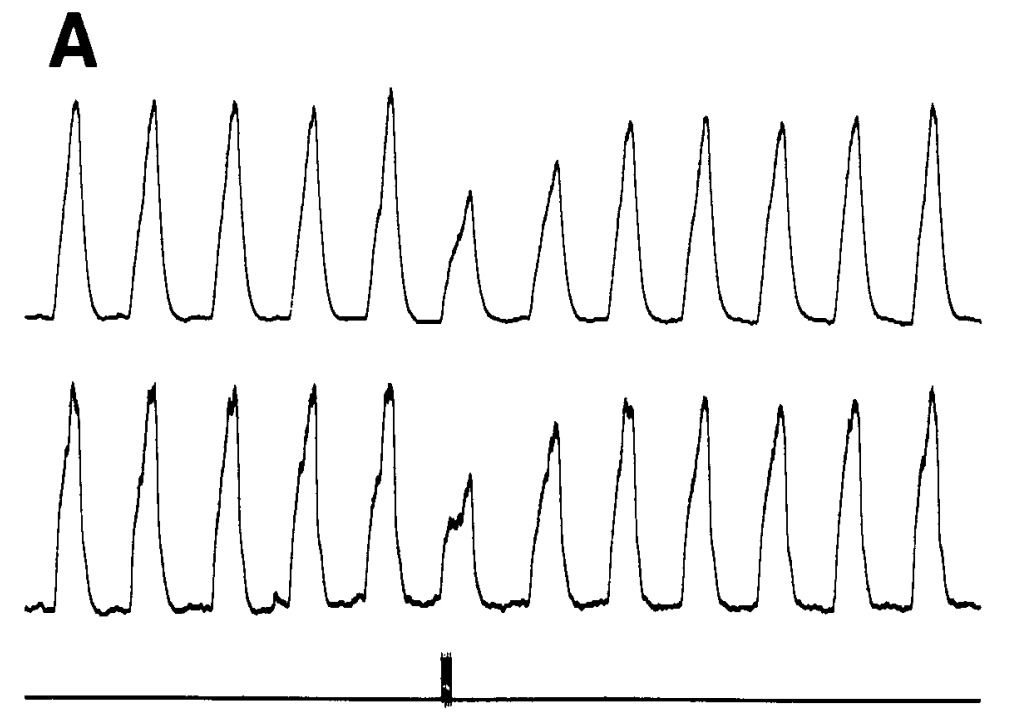

B
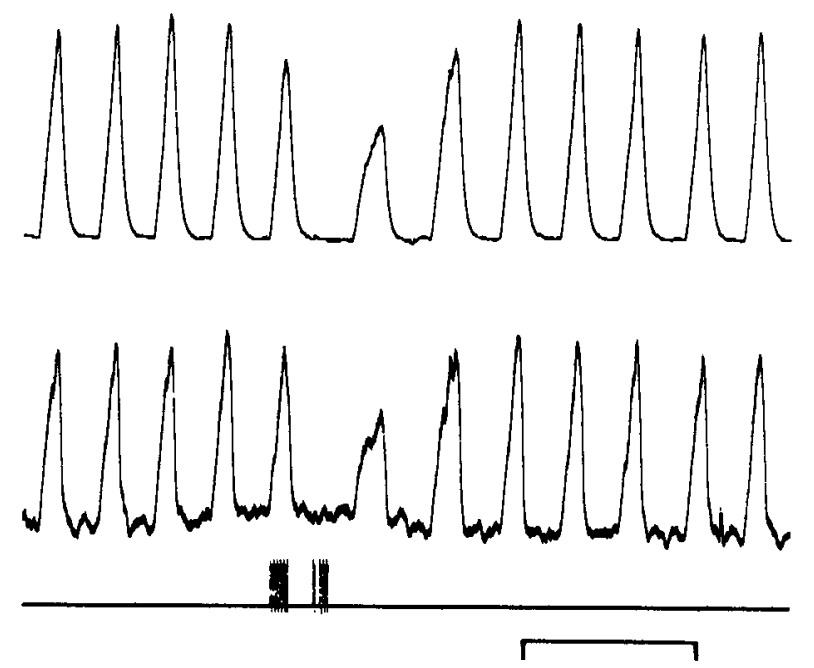

20 Seconds

Figure 5. Decreases in phrenic motoneuronal activity by injection of Glu in the ventral respiratory group. In each panel, top two traces are contralateral and ipsilateral phrenic nerve activity integrated with a Paynter filter; third trace indicates the pressure pulses causing ejection of 10 mм Glu. $A$, Glu, $4 \mathrm{nl}(40 \mathrm{pl})$ total, delivered in 4 pulses. The pipette was located $1.5 \mathrm{~mm}$ rostral and $4 \mathrm{~mm}$ lateral to the obcx. $B$, Glu, $10 \mathrm{~mm}(4$ $\mathrm{nl}, 4 \mathrm{pmol}$ ) delivered in each of 2 trains of pulses. The second pulse train was timed to begin $\approx 100$ msec before the expected inspiratory burst of phrenic motoneurons. Note the delay in onset of the following phrenic nerve discharge.

1964; Engberg et al., 1979a, b), which is possible but not likely. A given response could be reproduced 2 or 3 times in rapid succession with no noticeable decrement in response. In addition, the magnitude of a response was both volume- $(0.2-2.0$ $\mathrm{nl}$ ) and concentration- (1-10 mM) dependent, with no indication of a biphasic component suggestive of a depolarization block. Further, the decrease in phrenic nerve discharge due to DLH administration was qualitatively similar to that produccd by Glu, although DLH is less likely to produce a depolarizing block (Engberg et al., 1979a, b). In addition, local anesthesia induced by injections of 1-8 $\mathrm{nl}$ lidocaine prevented the response to Glu or NMDA but had little or no effect of its own on spontaneous phrenic nerve discharge.

The qualitative differences in responses obtained among the agonists may relate to the particular types of Glu receptors activated (Puil, 1981; Watkins and Evans, 1981). However, it is not possible on the basis of the present study to ascribe the responses to activation of a particular group of receptors. It is likely that various receptor types were activated. This is suggested by the qualitatively different responses elicited by NMDA and Glu, although a difference in efficacy of these agents at a particular set of receptors cannot be ruled out. The $2-3 \mathrm{~Hz}$ "bursting" elicited by NMDA, in contrast to the sustained increase in firing produced by Glu, may be similar to the receptorspecific responses of caudate neurons to NMDA and quisqualic acid (Herrling et al., 1983).

The effective spread of the Glu, NMDA, or DLH is difficult to gauge without direct measurement. Factors such as available space $(1 \mathrm{nl}=$ a sphere approximately $62 \mu \mathrm{m}$ in radius), pharmacokinetics, and the numbers and types of receptors on proximal neurons would affect the spread and responsiveness. The fact that tip displacements of $100-300 \mu \mathrm{m}$ could lead to noticeably different behavioral effects suggests a limited spread relative to space constants of the underlying mosaic of neuronal circuitry. This is supported on a theoretical basis by calculations showing that, $300 \mu \mathrm{m}$ from the site of a $10 \mathrm{nl}$ injection into brain tissue, the concentration of the injectate never exceeds
$20 \%$ of the injected concentration (Nicholson, 1985). The effect of inactivating mechanisms, such as active uptake into neurons or glia, would be expected to reduce the spread further. In addition, the responses to $1 \mathrm{~mm}$ Glu had a very rapid onset $(<200$ msec during inspiratory periods) and brief duration (Fig. $1 A, E$ ) before significant diffusion would have occurred (Nicholson, 1985). Further, a marked increase in neuronal impulse activity was recorded from a second electrode placed in the VRG $\approx 500$ $\mu \mathrm{m}$ from the ejecting tip, but there was no change in discharge rate recorded from an electrode placed outside of the VRG at an equivalent distance from the ejecting electrode. Although we cannot be certain that the cells outside the VRG were responsive to Glu, the simplest interpretation is that there was insufficicnt diffusion to activate these nonrespiratory cells. In contrast, VRG cells probably either had dendrites in the vicinity of the ejecting pipette or were synaptically activated.

We did not systematically compare the effects of Glu injection with electrical microstimulation. In one experiment, however, a tungsten microelectrode for electrical stimulation and a glass pipette for Glu were simultaneously placed in the excitatory region of the VRG. Proper positioning of the tungsten electrode was obtained using a specially designed stereotaxic system (Feldman and Cooke, 1982) and confirmed by the recording of respiratory neurons on both the glass and tungsten electrodes. While Glu ejection at this site always increased phrenic nerve discharge, electrical stimulation using pulse trains with a range of stimulus parameters $(10-100 \mu \mathrm{A}, 10-100 \mathrm{~Hz}, 0.1 \mathrm{msec}$ pulse duration) only reduced phrenic nerve discharge. The higher currents and stimulus frequencies abolished phrenic nerve discharge during a $10 \mathrm{sec}$ period of stimulation. Electrical stimulation, but not Glu injection, may have activated fibers of passage from inhibitory neurons that masked the excitatory response to activation of somatodendritic membranes. We previously reported that the short-latency $(\mathrm{msec})$ response to single electrical pulses was stereotyped throughout the VRG (and dorsal respiratory group) (Speck and Feldman, 1982). We also noted a variety of longer latency responses, including phase resetting, at 

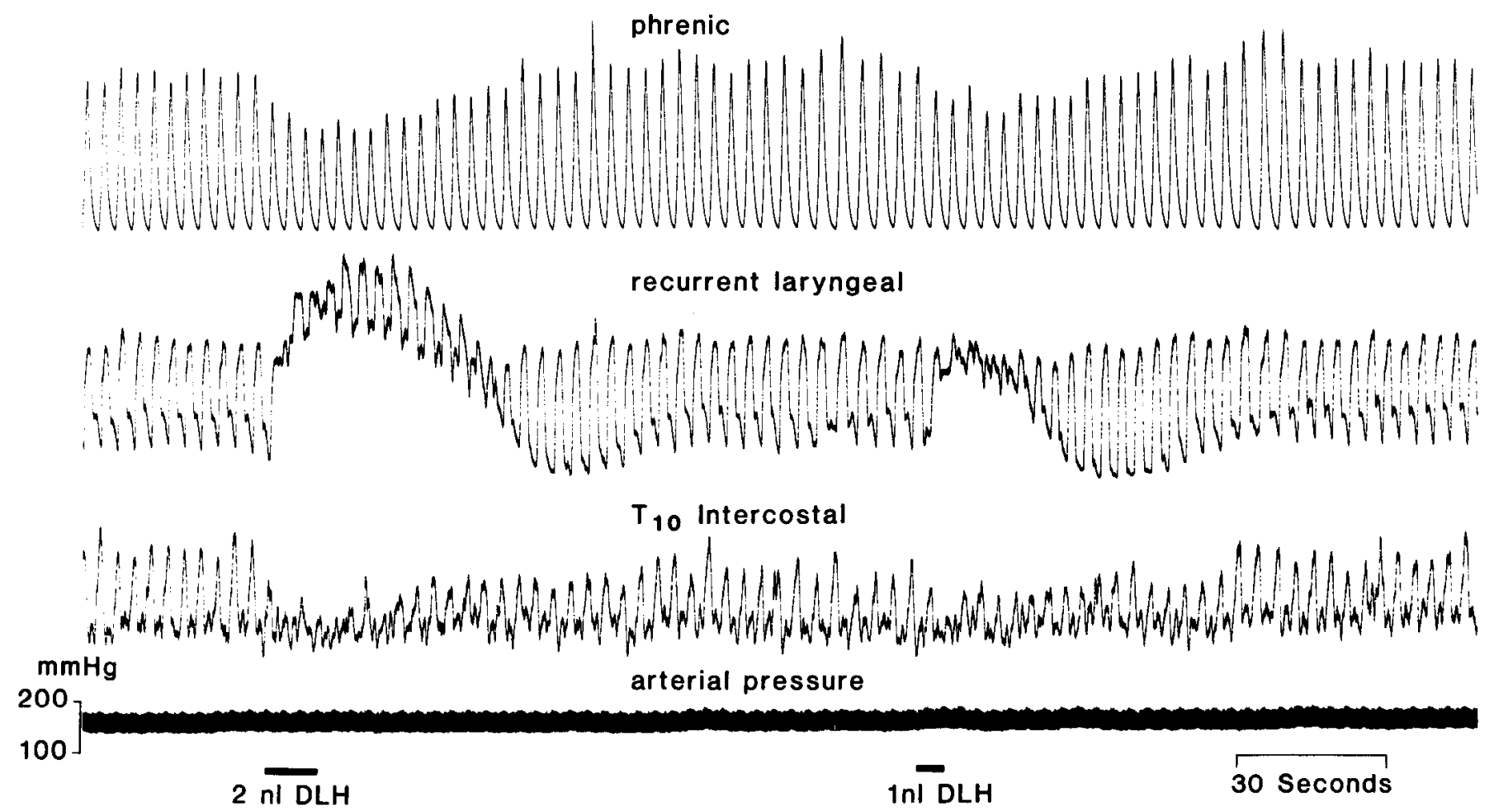

Figure 6. Effect on respiratory motor discharge of injecting DLH into the inhibitory region of the VRG (see Fig. 1). Traces (from the top) are integrated left phrenic nerve discharge (contralateral to injection site), integrated right recurrent laryngeal nerve discharge, integrated left internal intercostal nerve discharge, and arterial pressure. DLH, $10 \mathrm{~mm}$, injected $0.5 \mathrm{~mm}$ rostral and $3.0 \mathrm{~mm}$ lateral to the obex at the indicated times and volumes. Note, prior to DLH administration, the large-amplitude bursts on the recurrent laryngeal nerve occur during inspiration, with less discharge during expiration. DLH administration leads to a volume-dependent tonic discharge on which large bursts during expiration are superimposed. The discharge on the internal intercostal occurs largely during expiration and is decreased by DLH.

different sites in the VRG, but we did not analyze these responses in sufficient detail to determine the presence of an anatomical fine structure with respect to response types.

The depressor responses were qualitatively similar to those previously reported in response to Glu injections into the ventrolateral medulla of rat (Willette et al., 1983) or rabbit (Blessing and Reis, 1982). Nevertheless, there are important differences. We used much smaller volumes $(0.2-10$ vs $100-250 \mathrm{nl})$ and much lower total amounts of injected Glu $\left(2 \times 10^{-13}\right.$ to $10^{-11}$ vs $\geq 10^{-9} \mathrm{~mol}$ ). Thus, we were able to examine the microorganization of the neural circuitry underlying respiratory or cardiovascular function more closely. For example, even though we used much smaller injections of Glu with respect to both volume and total amount of drug injected, we obtained greater changes in blood pressure. While we cannot rule out species differences, the smaller response seen by others may result from diffusion of Glu into nearby regions that produce a pressor response (McCrimmon et al., 1986), thereby limiting the depressor response.

The relationship between the observed effects of local neuronal activation and the central respiratory function processed in these regions is affected by at least 2 considerations. First, since Glu receptors are considered ubiquitous on most neurons (Cotman et al., 1981), the stimulation is locally promiscuous. Thus, cells normally not activated in tandem can fire simultaneously. Second, the rates of firing of individual cells could be significantly greater than normal. Acknowledging these possibilities, we speculate that the transient bilateral changes in respiratory motor outflow (Figs. 2-5) are due to activation of neurons with somatodendritic membranes in the vicinity of the pipette that either directly or indirectly through interneurons alter the activity of other brain-stem respiratory neurons. The
VRG neurons directly activated by the excitatory amino acids may not be responsible for the generation of respiratory pattern themselves (Feldman and Speck, 1983; Speck and Feldman, 1982). Neither Glu antagonists nor lidocaine altered the rhythm of phrenic discharge, although the peak amplitude of phrenic nerve discharge was frequently decreased. Thus, the phase resetting of respiratory rhythm by injections of excitatory amino acids into either the excitatory or inhibitory regions of the VRG (Figs. 4, 5) may suggest the presence of a strong projection from neurons in these regions to the neurons responsible for generating respiratory rhythm.

The lack of a major effect of lidocaine or of Glu antagonists on phrenic nerve discharge suggests several hypotheses concerning the action of Glu and associated agonists on neurons initiating the observed responses:

1. Glu excites neurons, with somatodendritic membranes in the immediate vicinity of the pipette tip, that do not fire spontaneously in our experimental preparation. Thus, local anesthesia or Glu receptor blockade would have no effect. These cells may be active and important in other states, such as in the intact animal.

2. Glu greatly excites neurons that have low spontaneous rates of activity. Production of high rates of synchronous activity in a small cluster of neurons at a nodal point in the respiratory network may produce a noticeable perturbation; a small decrease in discharge rate, down to zero impulse activity induced by lidocaine or Glu receptor blockade, in this small group of cells may have little overall effect on the network.

3. Glu receptors are concentrated on the distal dendrites of cells mediating the response. If these cells are spontaneously active, then the depolarization from distal EPSP inputs may result in significantly increased neuronal activity. However, if 

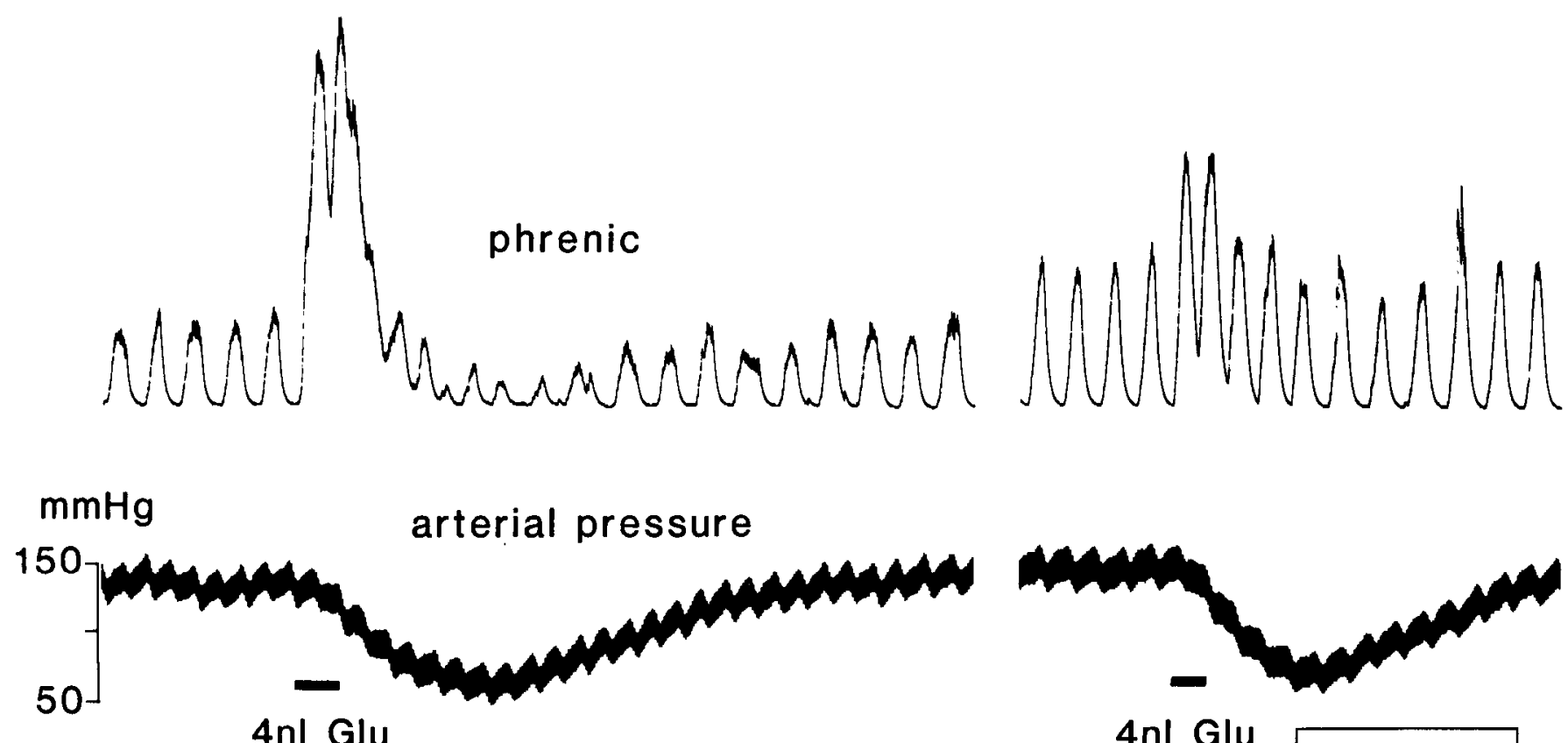

$4 \mathrm{nl}$ Glu

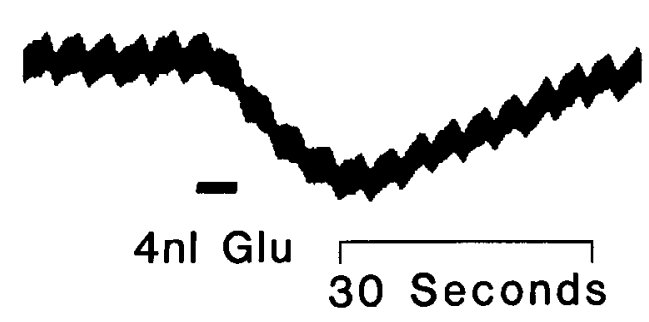

Figure 7. Effect of $10 \mathrm{~mm}$ Glu, injected into the excitatory region of the VRG, on phrenic nerve discharge and arterial pressure. Between panels the electrode was moved $300 \mu \mathrm{m}$ ventrally. Note the marked attenuation in the response of the phrenic nerve discharge (despite being shown at a greater gain), whereas the depressor response is similar at the 2 sites.

the distal dendritic input is not spontaneously active in this preparation, then lidocaine or Glu receptor blockade may have no noticeable effect on neuronal discharge rate generated by inputs onto other regions of the neuron.

Direct activation of bulbospinal inspiratory neurons may participate in producing the phrenic nerve excitation. However, this is unlikely to be the most important mechanism for producing the changes in respiratory timing or even the changes in amplitude of the integrated respiratory motoneuronal discharge for at least 2 reasons. First, the somas of bulbospinal neurons are distributed over a much wider region of the VRG (Cohen, 1979; Feldman, in press). This wider region encompasses the areas from which Glu injection inhibits or has no effect on phrenic nerve discharge. Second, we have previously shown that synchronous antidromic activation of bulbospinal neurons does not produce a resetting of phrenic rhythm (Feldman et al., 1984).

This is the first report suggesting a marked local heterogeneity in the brain-stem networks effecting respiratory pattern. A striking result of this study is that (sub)picomolar quantities of Glu, NMDA, or DLH, delivered in nanoliter volumes, produce sitespecific changes in respiratory motor nerve discharge that are readily detectable. The effects of even smaller injections or the injection of drugs with subtle effects are likely to be discernible with more sophisticated analysis. Finally, brain functions other than respiratory and cardiovascular (Feldman et al., 1985; Nelson et al., 1985) control may also have intrinsic properties that can be revealed by the nanoinjection technique. The use of this technique, in conjunction with appropriate drugs and physiological controls, could be an important tool in understanding local information transfer in brain and its consequences in terms of behavior.

\section{References}

Berman, A. L. (1968) The Brain Stem of the Cat, University of Wisconsin Press, Madison, WI.

Blessing, W. W., and D. J. Reis (1982) Inhibitory cardiovascular function of neurons in the caudal ventrolateral medulla of the rabbit:
Relationship to the area containing Al noradrenergic cells. Brain Res. 253: $161-171$.

Bradley, P. B., and A. P. Lucy (1983) Cholinoceptive properties of respiratory neurones in the rat medulla. Neuropharmacology 22:853858.

Cohen, M. I. (1979) Neurogenesis of respiratory rhythm in the mammal. Physiol. Rev. 59: 1105-1173.

Cotman, C. W., A. Foster, and T. Lanthorn (1981) An overview of glutamate as a neurotransmitter. In Glutamate as a Neurotransmitter, G. Di Chiara and G. L. Gessen, eds., pp. 1-27, Raven, New York.

Crawford, J. M., and D. R. Curtis (1964) The excitation and depression of mammalian cortical neurones by amino acids. Br. J. Pharmacol. 23: 313-329.

Crunelli, V., S. Forda, and J. S. Kelly (1985) Excitatory amino acids in the hippocampus: Synaptic physiology and pharmacology. Trends Neurosci. 8: 26-30.

Curtis, D. R., and R. W. Ryall (1966) Pharmacological studies upon spinal presynaptic fibres. Exp. Brain Res. 1: 195-204.

Dekin, M. S., G. B. Richerson, and P. A. Getting (1985) Thyrotropinreleasing hormone induces rhythmic bursting in neurons of the nucleus tractus solitarius. Science 229: 67-69.

Dev, N. B., and H. H. Loeschcke (1979) A cholinergic mechanism involved in the chemosensitivy of the medulla oblongata in the cat. Pfluegers Arch. 379: 29-36.

Dray, A., M. R. Hanley, R. D. Pinnock, and B. E. B. Sandberg (1983) A comparison of the release of substance $P$ and some synthetic analogues from micropipettes by microiontophoresis or pressure. Neuropharmacology 22: 859-863.

Engberg, I., J. A. Flatman, and J. D. C. Lambert (1979a) The actions of excitatory amino acids on motoneurones in the feline spinal cord. J. Physiol. (Lond.) 228: 227-261.

Engberg, I., J. A. Flatman, and J. D. C. Lambert (1979b) A comparison of extracellular and intracellular recording during extracellular microiontophoresis. J. Neurosci. Methods 1: 219-233.

von Euler, C. (1983) On the central pattern generator for the basic breathing rhythmicity. J. App. Physiol.: Respirat. Environ. Exercise Physiol. 55: 1647-1659.

Feldman, J. L. (in press) Neurophysiology of respiration in mammals. In Handbook of Physiology: The Nervous System, Intrinsic Regulatory Systems of the Brain. F. E. Bloom, ed., Williams \& Wilkins, Baltimore. 
Feldman, J. L., and J. E. Cooke (1982) A stereotaxic system for independent coordinated positioning of two or three microelectrodes. J. Neurosci. Methods 5: 139-146.

Feldman, J. L., and D. F. Speck (1983) Interactions among inspiratory neurons in dorsal and ventral respiratory groups in cat medulla. $J$. Neurophysiol. 49: 472-490.

Feldman, J. L., D. R. McCrimmon, and D. F. Speck (1984) Effect of synchronous activation of medullary inspiratory bulbo-spinal neurones on phrenic nerve discharge in cat. J. Physiol. (Lond.) 347: 241254.

Feldman, J. L., D. R. McCrimmon, H. L. Cohen, and D. O. Nelson (1985) Perturbation of cardiovascular function by picomoles of $\mathrm{L}-\mathrm{glu}$ tamate injected into rat brainstem. Fed. Proc. 44: 1554.

Goodchild, A. K., R. A. L. Dampney, and R. Bandler (1982) A method for evoking physiological responses by stimulation of cell bodies, but not axons of passage, within localized regions of the central nervous system. J. Neurosci. Methods 6: 351-363.

Hedner, J., T. Hedner, P. Wessberg, and J. Jonason (1983) Effects of TRH and TRH analogues on the central regulation of breathing in the rat. Acta Physiol. Scand. 117: 427-437.

Herrling, P. L., R. Morris, and T. E. Salt (1983) Effects of excitatory amino acids and their antagonists on membrane and action potentials of cat caudate neurones. J. Physiol. (Lond.) 339: 207-222.

Kalia, M., J. L. Feldman, and M. I. Cohen (1979) Afferent projections to the inspiratory neuronal region of the ventrolateral nucleus of the tractus solitarius in the cat. Brain Res. 171: 135-141.

LaBossiere, E. (1976) Histological Processing for the Neural Sciences, Thomas, Springfield, IL.

Lipski, J., and E. G. Merrill (1980) Electrophysiological demonstration of the projection from expiratory neurones in rostral medulla to contralateral dorsal respiratory group. Brain Res. 197: 521-524.

McCrimmon, D. R., J. L. Feldman, D. F. Speck, H. H. Ellenberger, and J. C. Smith (1986) Functional heterogeneity of dorsal, ventral and pontine respiratory groups revealed by micropharmacological techniques. In Neurobiology of the Control of Breathing, 10th Nobel Con- ference, C. von Euler and H. Lagercrantz, eds., Raven, New York (in press).

Merrill. E. G., and L. Fedorko (1984) Monosynaptic inhibition of phrenic motoneurons: A long descending projection from Bötzinger neurons. J. Neurosci. 4: 2350-2353.

Morin-Surun, M. P., J. Champagnat, M. Denavit-Saubié, and S. Moyanova (1984) The effects of acetylcholine on bulbar respiratory related neurones. Naunyn Schmiedebergs Arch. Pharmacol. 325: 205208

Nelson, D. O., J. L. Feldman, D. R. McCrimmon, and H. L. Cohen (1985) Cardiovascular function is markedly altered by picomoles of L-glutamate injected into rat brainstem. Soc. Neurosci. Abstr. 11. 191

Nicholson, C. (1985) Diffusion from an injected volume of a substance in brain tissue with arbitrary volume fraction and tortuosity. Brain Res. 333: 325-329.

Palmer, M. R., S. M. Wuerthele, and B. J. Hoffer (1980) Physical and physiological characteristics of micropressure ejection of drugs from multibarreled pipettes. Neuropharmacology 19: 931-938.

Puil, E. (1981) S-Glutamate: Its interactions with spinal neurons. Brain Res. Rev. 3: 229-322.

Speck, D. F., and J. L. Feldman (1982) The effects of microstimulation and microlesions in the ventral and dorsal respiratory groups in medulla of cat. J. Neurosci. 2: 744-757.

Watkins, J. C., and R. H. Evans (1981) Excitatory amino acid transmitters. Annu. Rev. Pharmacol. Toxicol. 21: 165-204.

Welzl, H., T. R. Tolle, and J. P. Huston (1985) Intracranial application of substances in the unrestrained, awake rat by pressure injection through glass micropipettes. J. Neurosci. Methods 13: 1-8.

Willette, R. N., P. P. Barcas, A. J. Krieger, and H. N. Sapru (1983) Vasopressor and depressor areas in the rat medulla. Identification by microinjection of L-glutamate. Neuropharmacology 22: 1071-1079.

Zieglgansberger, W., and E. A. Puil (1973) Actions of glutamic acid on spinal neurones. Exp. Brain Res. 17: 35-49. 